\title{
Metal-Organic Frameworks: o que são e para que servem
}

\author{
Filipe A. Almeida Paz, João Rocha \\ Departamento de Química, CICECO, Universidade de Aveiro \\ rocha@ua.pt
}

\begin{abstract}
Introducing Metal-Organic Frameworks and their Applications - This paper presents, in a simple way, the crystalline solids known as Metal-Organic Frameworks (MOFs). The basic principles employed in the design and construction of MOFs are summarized, from the selection of the metallic centers and organic linkers (ligands) to the presentation of the most common synthetic methods and experimental conditions. A number of properties and potential applications of these materials are discussed, such as permanent nanoporosity, light emission and use in sensors, storage and separation of gases, and heterogeneous catalysis. A brief overview of the research carried out in Portugal in this new area of coordination chemistry is presented, emphasizing the studies performed at the University of Aveiro.
\end{abstract}

De forma muito simples, apresentam-se os sólidos conhecidos como redes metalo-orgânicas ou, em inglês, "MetalOrganic Frameworks” (MOFs). Resumem-se as ideias básicas usadas no design e na construção de MOFs, desde a selecção dos centros metálicos e das moléculas (ligandos) orgânicas até aos métodos e às condições de síntese mais comuns. Discutem-se algumas das propriedades e potenciais aplicações destes materiais (nomeadamente, nanoporosidade permanente, emissão de luz e uso em sensores, armazenamento e separação de gases, catálise heterogénea). Dá-se, ainda, uma breve panorâmica da investigação feita em Portugal nesta nova área da química de coordenação enfatizando, em particular, o trabalho desenvolvido na Universidade de Aveiro.

\section{INTRODUÇÃO}

Tal como outros ramos da Ciência, a Química evoluiu ao longo dos tempos impulsionada por descobertas disruptivas que abriram novas áreas do conhecimento. Um exemplo marcante é a síntese acidental da ureia por Friedrich Wöhler, que mudou o paradigma vigente segundo o qual apenas os seres vivos seriam capazes de produzir moléculas orgânicas, baseadas em ligações covalentes [1]. De forma igualmente notável, no final do século XIX, Alfred Werner descreveu pela primeira vez a composição e geometria de complexos de coordenação nos quais os centros metálicos se ligam a moléculas ou iões que os rodeiam. O contributo de Werner foi de tal maneira marcante que lhe valeu em 1913 o Prémio Nobel da Química e a memória futura de fundador da química de coordenação [2]. Mais recentemente, Jean-Marie Lehn (Nobel da Química de 1987) demonstrou a enorme importância que assumem nos sistemas químicos, em particular biológicos, as interacções supramoleculares, que compreendem, em geral, ligações de baixa energia (pontes de hidrogénio, por exemplo).

A Química Inorgânica, em particular a área da química de coordenação, recebeu na última década e meia um novo ímpeto criativo com a síntese e o estudo da estrutura e das propriedades dos polímeros de coordenação, frequentemente denominados redes metalo-orgânicas (do inglês "Metal-Organic Frameworks" - MOFs). Apesar de uma enorme comunidade química investigar neste domínio, alguns dos principais avanços devem-se aos grupos de investigação liderados por Omar Yaghi (Universidade de Berkeley, EUA) [3], Susumu Kitagawa (Universidade de Quioto, Japão) [4] e Gérard Férey/Cristian Serre (Instituto
Lavoisier, Universidade de Versalhes, França) [5]. Apesar de serem conhecidas estruturas de MOFs desde meados do século Xx, Yaghi foi o primeiro a demonstrar em 1999, usando o MOF-5 como exemplo (Figura 1), que a síntese destes compostos poderia ser racionalizada e controlada, de forma a dar origem a compostos com nanoporosidade permanente e com significativa robustez química e mecânica [6]. Yaghi foi também pioneiro ao descrever a síntese de materiais isotípicos (isto é, possuindo a mesma conectividade de unidades básicas) cujo tamanho de poro está sob o rigoroso controlo do investigador. Yaghi capturou a essência das suas ideias ao cunhar o domínio "química reticular" (Figura 2) [7].

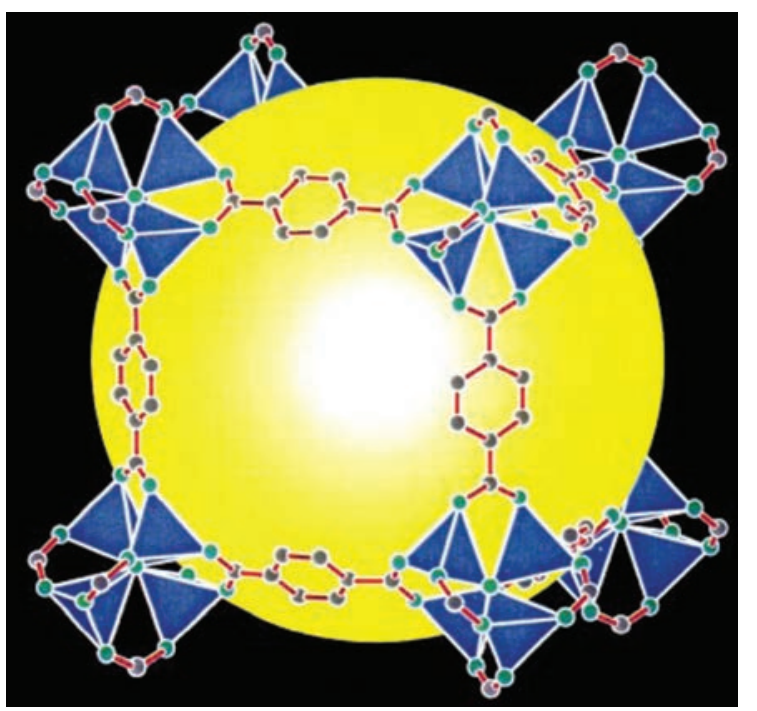

Figura 1 - Célula cúbica primitiva do MOF-5 enfatizando as pontes de tereftalato entre agregados de óxido de zinco. Figura adaptada com permissão [6]. (Direitos de autor 1999 Macmillan) 

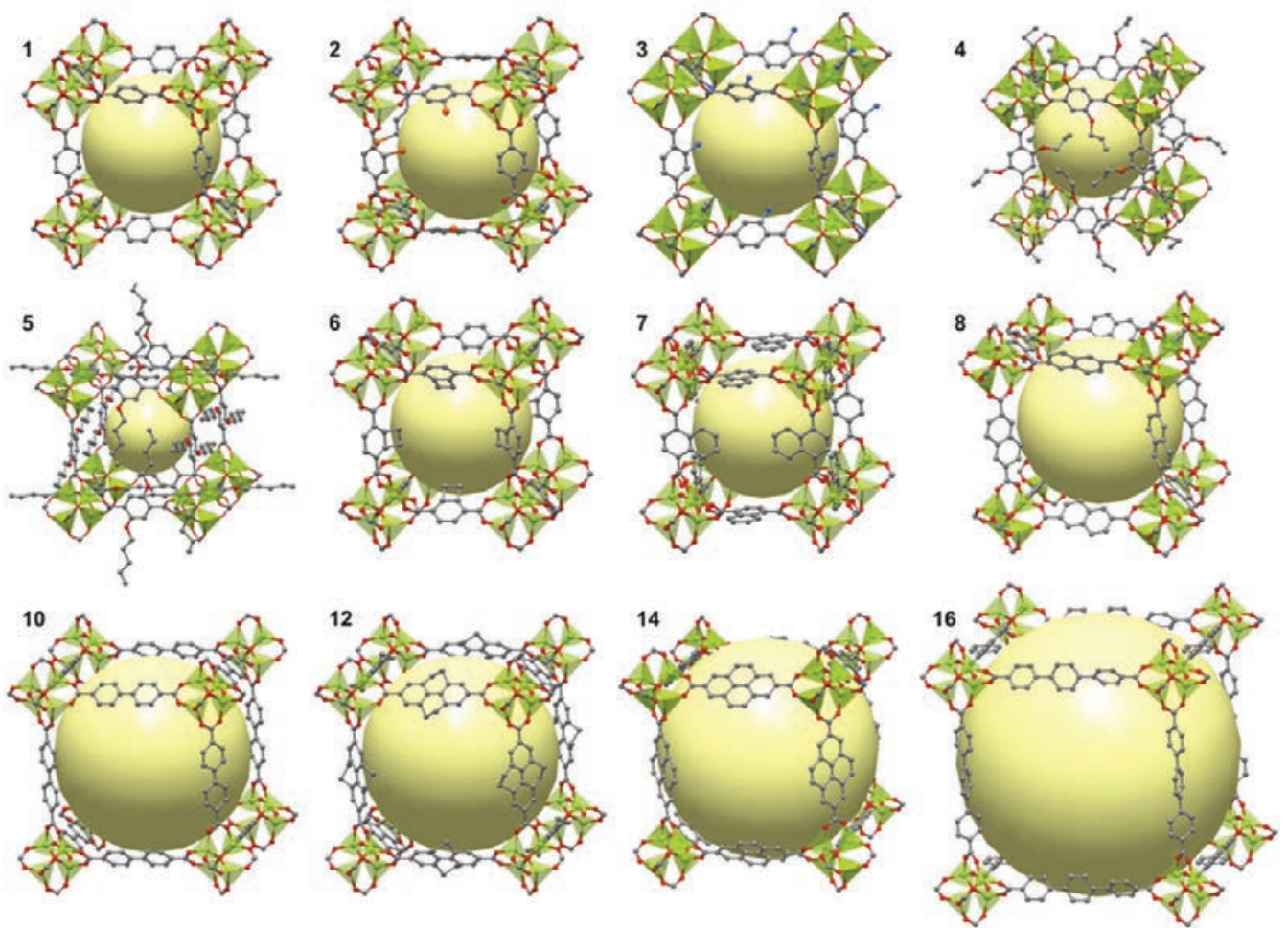

Figura 2 - Estruturas cristalinas da série IRMOF- $n$ de materiais porosos (IRMOF = Iso-Reticular Metal-Organic Framework). A série de compostos é preparada usando moléculas orgânicas derivadas do ácido tereftálico de forma a aumentar o tamanho da ponte entre agregados de óxido de zinco mantendo a topologia cúbica da rede, tal como no MOF-5 (ver Figura anterior). (Direitos de autor 2013 [8] Patrícia Silva)

\section{COMO CONSTRUIR UM MOF?}

\subsection{UNIDADES BÁSICAS DE CONSTRUÇÃO}

A formação destes compostos cristalinos pode ser compreendida considerando duas unidades básicas de construção (por vezes chamadas unidades primárias de construção), os nós e as pontes, o que permite, também, utilizar certos conceitos matemáticos (nomeadamente topológicos) no design de novos materiais (Figura 3). As pontes são, em geral, moléculas orgânicas (ligandos) relativamente rígidas, contendo pelo menos dois grupos funcionais capazes de coordenar os iões metálicos e localizados em posições exo, de forma a promover a polimerização. Os nós são formados por um ião (centro) metálico ou por cachos (agregados) de iões metálicos possuindo posições de coordenação disponíveis para se ligarem às pontes. Dependendo das condições reaccionais utilizadas, podem obter-se vários compostos (ou redes) para a mesma estequiometria de nós e pontes, sendo que a dimensionalidade final pode também variar (Figura 3).

\subsection{ESCOLHA DAS UNIDADES BÁSICAS DE CONSTRUÇÃO}

\subsubsection{AS PONTES: LIGANDOS ORGÂNICOS}

Será que qualquer molécula orgânica serve para construir um MOF? A resposta curta é: não. As moléculas orgânicas, cuja função estrutural é interligar os centros metálicos, devem satisfazer um conjunto de requisitos a fim de poderem dar origem a MOFs. Em primeiro lugar, devem conter grupos funcionais (idênticos ou não) capazes de coordenar os centros metálicos escolhidos, de preferência em posições exo (opostas na molécula) de modo a formar pontes. Os ligandos mais utilizados para este propósito são os ácidos carboxílicos, grupos hidroxilo, ácidos sulfónicos e fosfónicos e anéis contendo heteroátomos (em particular azoto) (Figura 4). As moléculas orgânicas devem também, de preferência, ser razoavelmente rígidas, o que se traduz no uso frequente de ligandos com anéis aromáticos. Esta "rigidez estrutural” dos ligandos permite, por um lado, reduzir a ocorrência de isomerismo supramolecular, e por outro lado, promover a formação de redes robustas e estáveis.

\subsubsection{OS NÓS: CENTROS METÁLICOS}

Uma selecção judiciosa dos centros metálicos é fundamental na preparação dos MOFs, não só porque determina muitas das propriedades finais destes (tais como magnetismo, fotoluminescência, catálise), mas também porque precisa a dimensionalidade (1D-3D) do MOF. Após mais de duas décadas de intensa investigação, um grande número de metais da tabela periódica foi já utilizado na construção de MOFs. De facto, os metais de transição (MT), geralmente da primeira série, assim como os metais lantanídeos (Ln) são os mais utilizados. Do ponto de vista estrutural, os nós baseados em MT e Ln apresentam conectividades distintas: enquanto os primeiros assumem tipicamente números de coordenação entre 2 e 6 (Figura 5), os segundos exibem geometrias de coordenação que acomodam até 12 átomos (Figura 6), sendo bastante raros os números de coordenação inferiores a 6. As geometrias de coordenação dos MT são geralmente rígidas (limitada variação dos comprimentos e ângulos de ligação) contrastando com a maior flexibilidade observada com os iões Ln. Do ponto de vista topológico, os 


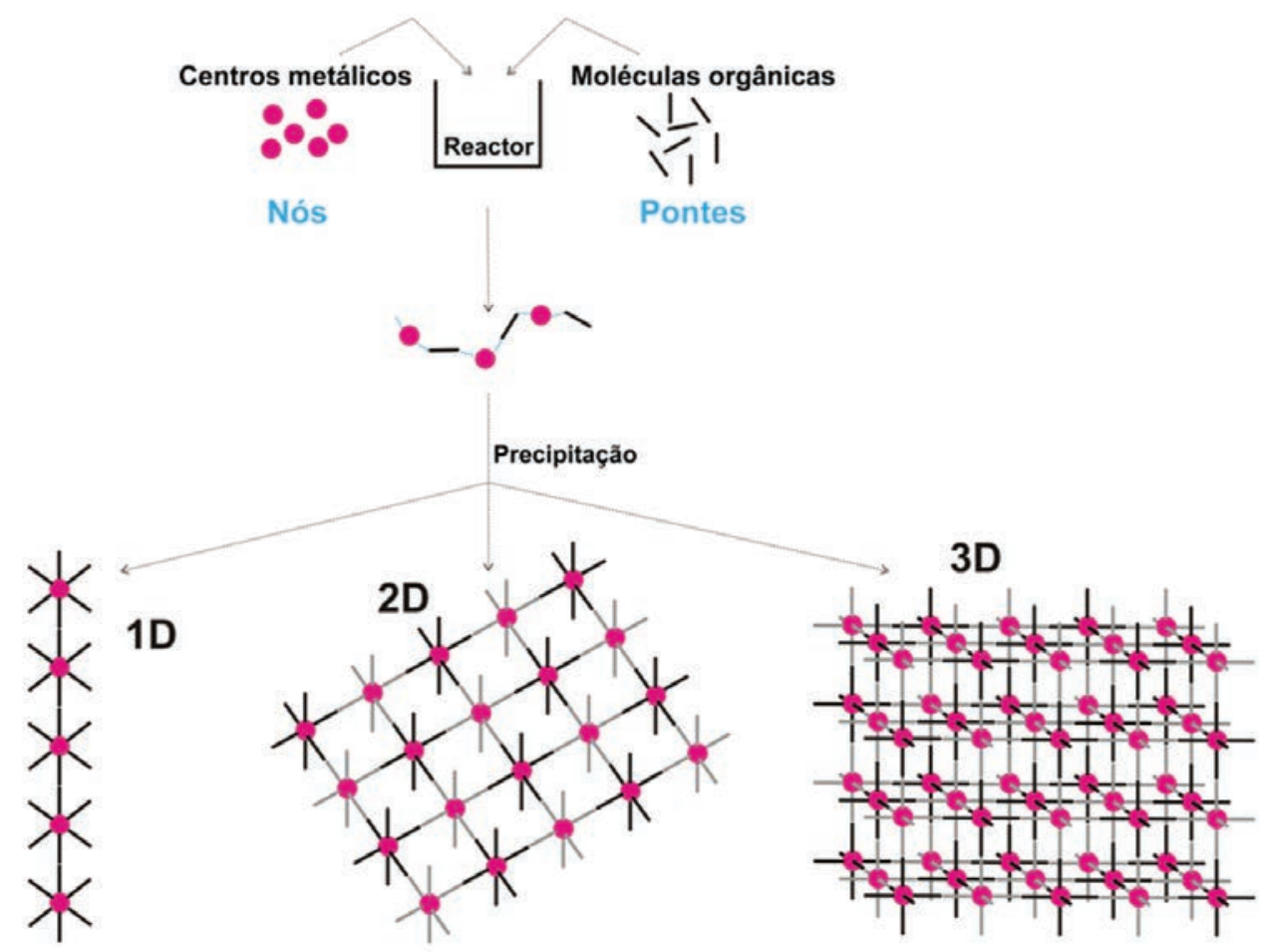

Figura 3 - Construção de MOFs a partir de unidades básicas: a interligação dos centros metálicos (nós) através de ligandos orgânicos (pontes) forma redes de dimensionalidade variável (1D - 3D). (Direitos de autor 2013 [8] Patrícia Silva)

Grupos dadores de oxigénio

Figura 4 - Grupos funcionais mais comuns usados na construção de MOFs. Figura adaptada com permissão. (Direitos de autor 2013 [9] Sérgio M. F. Vilela)

MOFs baseados em MT e Ln são bastante diferentes exibindo estes, frequentemente, redes muito complexas. Apesar de entre os iões MT e Ln se observar uma grande variabilidade de raios iónicos, os últimos apresentam geralmente o estado de oxidação formal +3 , enquanto que para os primeiros são conhecidos compostos com iões em diversos estados de oxidação. Por outro lado, os Ln coordenam-se também preferencialmente a átomos dadores de grande electronegatividade (tal como o oxigénio) enquanto que com MT a ocorrência de ligandos azotados é mais frequente.

\subsection{SíNTESE QUÍMICA}

As condições específicas em que as várias unidades primárias de construção (nós e pontes) reagem formando MOFs podem variar consideravelmente, merecendo uma particular atenção.

\subsubsection{CONDIÇÕES REACCIONAIS}

É frequente encontrar descrito na literatura que a estrutura dos MOFs obtidos e a pureza destes dependem de certos parâmetros experimentais, nomeadamente:

i) Fonte de metal: os centros metálicos podem ser oriundos de vários tipos de reagentes (“fontes”), nomeadamente sais (nitratos, cloretos, acetatos, etc.) e compos-

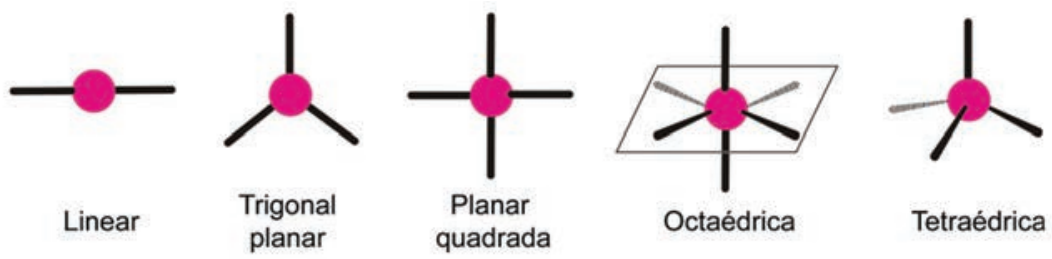

Figura 5-Modos de coordenação mais representativos encontrados em MOFs baseados em metais de transição (números de coordenação compreendidos entre 2 e 6), em particular, da primeira série. (Direitos de autor 2013 [8] Patrícia Silva) 


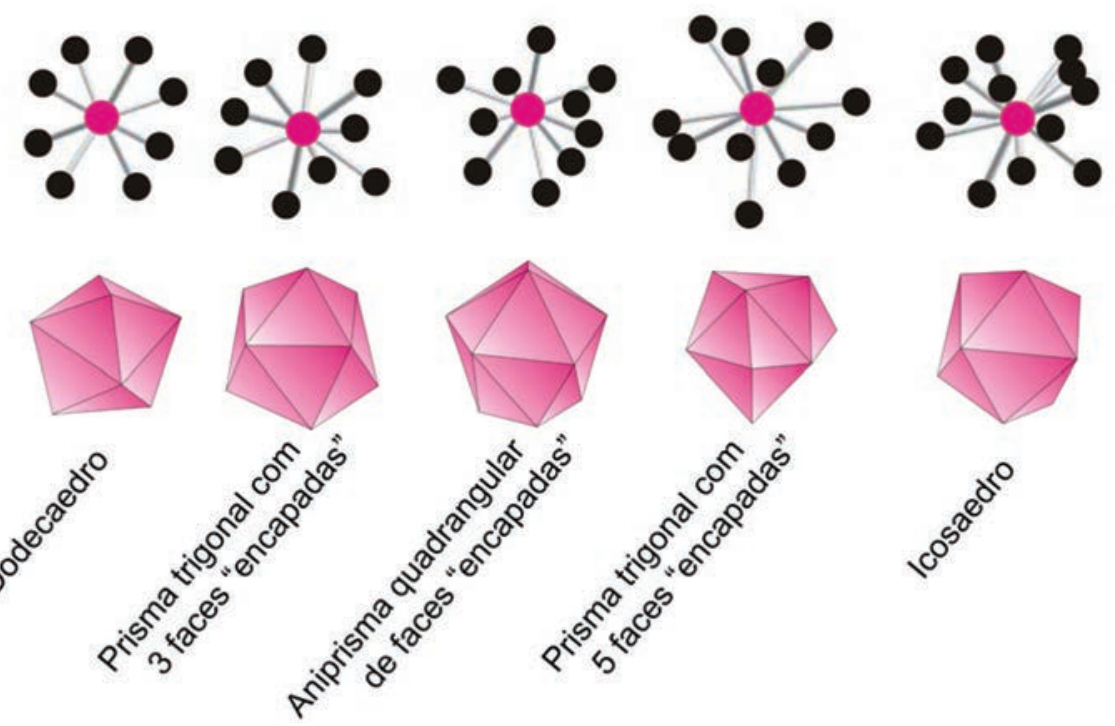

Figura 6 - Modos de coordenação mais representativos encontrados em MOFs baseados em iões lantanídeo. (Direitos de autor 2013 [8] Patrícia Silva)

tos inorgânicos, como óxidos metálicos; a selecção da fonte escolhida está relacionada com a sua solubilidade no solvente utilizado, reactividade e estado de oxidação pretendido;

ii) Solvente: de acordo com o método escolhido (ver em baixo), poderá ter de dissolver completamente tanto as pontes como os nós; em geral, os solventes mais utilizados são a água e a dimetilformamida, mas o etanol, metanol ou sulfóxido de dimetilo (ou misturas destes) são também muito utilizados;

iii) $p H$ : em geral, é controlado pela adição de ácidos ou bases (por exemplo, $\mathrm{HCl}$ ou $\mathrm{NaOH}$ ) de forma a controlar o grau de protonação das moléculas orgânicas;

iv) Composição da mistura reaccional: as razões molares utilizadas na mistura reaccional, envolvendo os nós (centros metálicos), as pontes (moléculas orgânicas) e o solvente desempenham um papel fundamental na diversidade estrutural obtida nas sínteses;

iv) Factores relacionados com o método de síntese: factores como a temperatura, o modo como a temperatura máxima é atingida, a presença ou ausência de rampas de aquecimento ou arrefecimento, a pressão (ambiente, induzida ou auto-gerada) e o tempo de reacção (desde alguns minutos até semanas) determinam, também, o tipo de materiais obtidos e a sua pureza e cristalinidade.

\subsubsection{MÉTOdOS DE SÍNTESE}

De acordo com os objectivos de cada grupo de investigação, são vários os métodos de síntese utilizados na preparação de MOFs. Em geral, privilegia-se a preparação de materiais muito cristalinos, de preferência na forma de monocristais de dimensão adequada para a resolução estrutural por difracção de raios X. São, também, utilizados métodos rápidos e com menor formação de resíduos, tal como o aquecimento por micro-ondas, com o objectivo de escalar as sínteses tornando-as mais atractivas para aplicações industriais. i) Evaporação lenta e/ou difusão de solventes. Permite obter materiais muito cristalinos, tendo sido o primeiro método utilizado nesta área de investigação. Colocam-se em contacto soluções concentradas das unidades de construção (pontes e nós), resultando a difusão lenta de um meio no outro meio na cristalização dos novos materiais. Sendo muito simples e eficaz este método é, contudo, lento (dias a meses) e oferece rendimentos muito baixos;

ii) Síntese solvotérmica. Neste método, originalmente desenvolvido para a preparação de zeólitos, coloca-se a mistura reaccional num reactor (geralmente de aço revestido internamente a Teflon), que é aquecido a temperaturas superiores às do ponto de ebulição do solvente, evoluindo a síntese sob condições de pressão auto-gerada, com ou sem agitação. Rampas variáveis de aquecimento ou arrefecimento são também comuns, tendo em vista promover o crescimento dos cristais. Este é o método de eleição para a preparação de MOFs, podendo os tempos de reacção variar de algumas horas a vários dias;

iii) Síntese assistida por micro-ondas. Com o aparecimento de fornos de micro-ondas de bancada, este método tem vindo a ganhar terreno na síntese de MOFs. Em vez do aquecimento por convecção da convencional síntese solvotérmica em reactor fechado, este método utiliza micro-ondas para um aquecimento mais rápido e uniforme do sistema reaccional. É um método bastante simples, caracterizado por uma grande eficácia no aquecimento e que reduz a produção de resíduos e produtos secundários. Tal como descrito numa revisão feita pelo grupo de Aveiro [10], a simples variação dos parâmetros inerentes a este método permite um maior controlo do tamanho e hábito dos cristais, e um aumento da selectividade da síntese no sentido da obtenção do material desejado;

iv) Síntese ionotérmica. Esta variante que pode ser baseada tanto na síntese tradicional solvotérmica como na síntese assistida por micro-ondas, utiliza como 
meio reaccional líquidos iónicos ou misturas eutécticas. Aqueles funcionam simultaneamente como solventes, moléculas estruturantes das redes ou como contra-iões, possuindo a vantagem de serem considerados solventes verdes;

v) Síntese electroquímica. Baseia-se na transferência de electrões entre um eléctrodo e o electrólito ou espécies em solução. Não sendo um método muito usado na síntese "académica” de MOFs prima pelo facto de ter sido desenvolvido pela BASF para a síntese em grande escala do MOF HKUST-1, uma rede 3D formada por iões $\mathrm{Cu}^{2+}$ e por ácido benzeno-1,3,5-tricarboxílico (Figura 7). Para uma imagem do dispositivo experimental usado neste método de síntese aconselha-se a consulta do artigo de revisão de Mueller e colaboradores [11];

vi) Síntese assistida por ultra-sons. É um dos métodos menos explorados, em que o aquecimento pontual é devido à criação e ao crescimento e colapso da cavitação acústica. Estima-se que a temperatura pontual alcance valores entre 5000 e 25000 K. Na síntese de MOFs, a ultrassonicação promove uma nucleação homogénea, com tempos de cristalização muito curtos, sendo essencialmente utilizada para a fabricação de nanocristais, tal como descrito para o icónico MOF-5 [12];

vii) Síntese “one-pot”. Não sendo um método de uso geral na preparação de MOFs é extremamente simples, consistindo na agitação (magnética ou mecânica) de soluções das unidades de construção (nós e pontes) à temperatura ambiente e pressão atmosférica. Este método tem conquistado adeptos nos últimos anos pela sua rapidez e pelo seu baixo consumo energético, permitindo obter nanocristais [13];

viii) Mecanossíntese. Este método baseia-se na utilização de energia mecânica para promover a reacção entre as unidades de construção (nós e pontes) na ausência de solvente. Apesar de ser um método muito explorado em química orgânica, raramente tem sido descrito para a síntese de MOFs [14];

ix) Spray-Drying. Sendo um dos mais recentes métodos não é surpreendente encontrar na literatura apenas um punhado de descrições de casos de sucesso [15]. Baseia-se simplesmente na obtenção de um pó cristalino pela rápida evaporação do solvente de uma mistura reaccional através da utilização de um gás de arrasto quente. Este método parece resolver muitos dos problemas subjacentes aos métodos anteriores no respeitante à preparação de nanocristais de MOFs com uma distribuição uniforme de cristalite.

\section{MOFS FUNCIONAIS}

Para além das inúmeras, variadas e esteticamente apelativas arquitecturas adoptadas pelos MOFs, que só por si constituem um estimulante campo de investigação, existe um grande interesse em explorar a relação simbiótica entre os centros metálicos e as moléculas orgânicas por forma a conceber e preparar de forma racional novos materiais funcionais. As propriedades desejadas podem ter origem nos centros metálicos (por exemplo, fotoluminescência ou magnetismo), nos ligandos orgânicos (por exemplo, reactividade química, que permite reacções de pós-síntese a ter lugar nos nanocanais) ou resultarem da interacção entre ambos.

\subsection{POROSIDADE}

De entre todas as propriedades dos MOFs, a presença de nanoporosidade permanente é indiscutivelmente a mais procurada e estudada. De facto, a preparação de materiais porosos abre a possibilidade da sua utilização numa vasta gama de aplicações, tais como: i) armazenamento de espécies químicas (nomeadamente, metano ou hidrogénio); ii) separação de gases (por exemplo, retenção de $\mathrm{CO}_{2}$ de uma mistura de gases naturais); iii) actividade catalítica devida a uma fácil acessibilidade dos substratos aos centros activos; iv) capacidade de acomodar moléculas com interesse biológico, por exemplo, fármacos. A preparação de MOFs porosos não é, no entanto, uma tarefa inteiramente trivial. O simples aumento da dimensão dos ligandos-ponte não garante a obtenção de materiais muito porosos, já que as estruturas podem colapsar ou pode ocorrer interpenetração de redes idênticas.

\subsubsection{Armazenamento de metano e hidrogénio}

O metano (componente do gás natural) e o hidrogénio são considerados alternativas energéticas "limpas", relativamente aos combustíveis fósseis, sendo imperativo desenvolver materiais baratos que os armazenem e libertem controladamente, de forma simples, segura e eficiente. $\mathrm{O}$ Departamento de Energia dos Estados Unidos enunciou, recentemente, as metas a atingir no desenvolvimento de novos materiais com aplicações nesta área: para o hidrogénio a temperaturas próximas da ambiente e pressões inferiores a 100 bar, uma percentagem de armazenamento de $9,9 \%$ em massa e $81 \mathrm{~g} \mathrm{dm}^{-3}$; para o metano o valor situa-se nos $180 \mathrm{~cm}^{3} \mathrm{~cm}^{-3}$ em condições normais de pressão e temperatura. $\mathrm{O}$ maior problema colocado pelo metano e hidrogénio é a sua fraca interacção com os materiais utilizados. Até à data, o MOF comercial HKUST-1 (Figura 7) é aquele que apresenta a maior capacidade de armazenamento de metano (cerca de $230 \mathrm{~cm}^{3} \mathrm{~cm}^{-3}$ a 35 bar e $270 \mathrm{~cm}^{3} \mathrm{~cm}^{-3}$ a 65 bar) [50].

Chegou-se à conclusão que o armazenamento de hidrogénio exige dois requisitos fundamentais: i) nanoporos permanentes de tamanho reduzido; ii) grupos funcionais capazes de interagir quimicamente com o hidrogénio e, assim, estabilizar as moléculas nos canais (por exemplo, ligações duplas ou átomos polarizáveis). Um dos trabalhos nesta área baseia-se na modificação pós-síntese de álcoois para alcóxidos metálicos. Este trabalho do grupo de investigação de Joseph Hupp da Universidade Northwestern (Illinois, EUA) [51] demonstra que tal procedimento aumenta a capacidade de adsorção para 1,32\% em massa (a 77K e pressão de $1 \mathrm{~atm}$ ). 


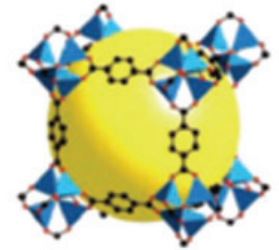

MOF-5

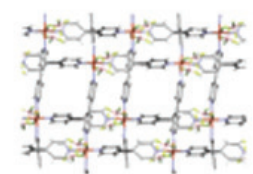

ELM-11

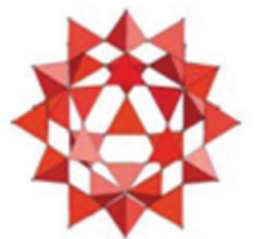

Cr-MIL-100

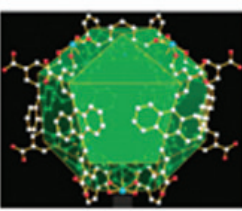

PCN-14

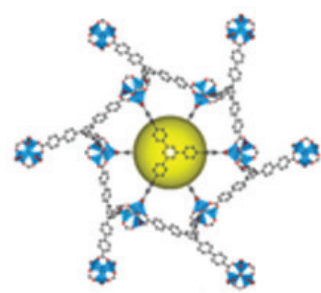

MOF-200

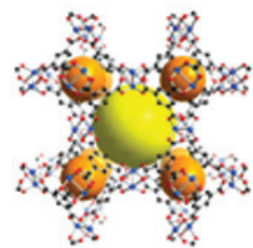

HKUST-1

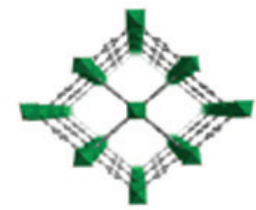

MIL-47

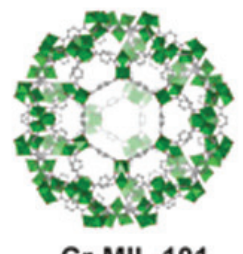

Cr-MIL-101

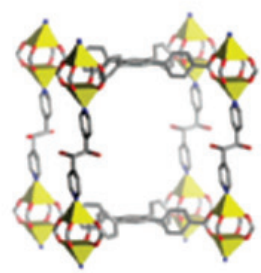

DO-MOF

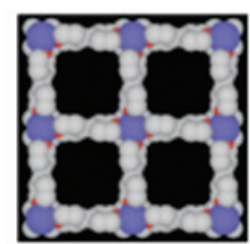

[CuSiF $\left(4,4^{\prime}-\right.$-bpy $\left.)\right]$

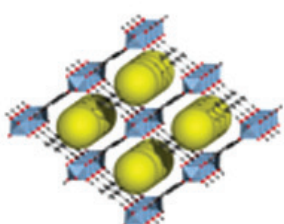

MIL-53

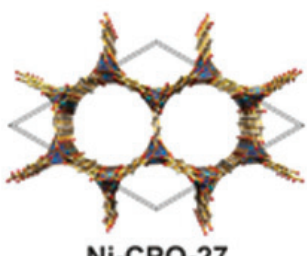

$\mathrm{Ni}-\mathrm{CPO}-27$

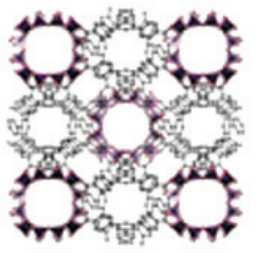

$\left[\mathrm{Be}_{12}(\mathrm{OH})_{12}(\mathrm{BTB})_{4}\right]$

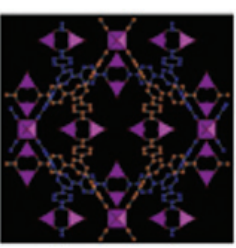

MOF-14

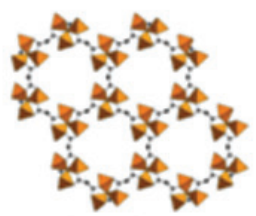

MIL-88

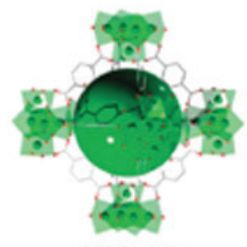

UiO-66

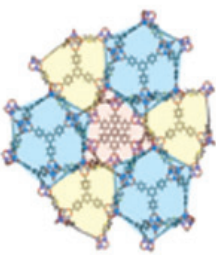

UMCM-2

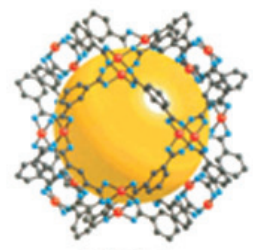

MOP-1

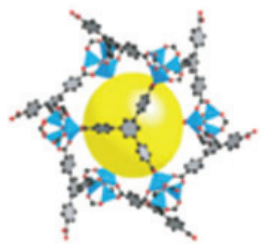

MIL-177
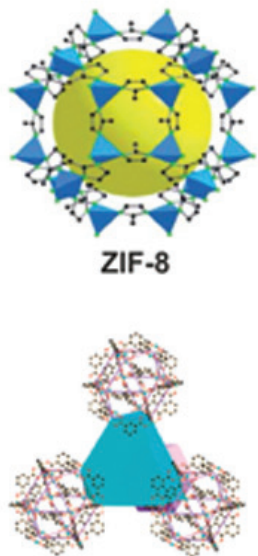

NOTT-116
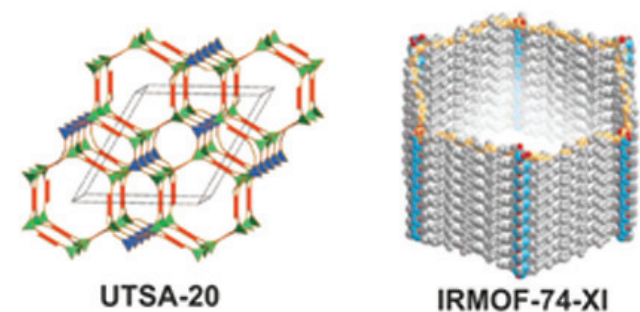

IRMOF-74-XI

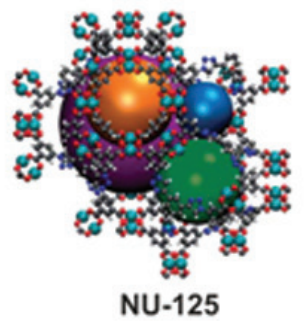

Figura 7 - Alguns MOFs porosos descritos na literatura especializada [16-49]

\subsubsection{Armazenamento de dióXIDO de CARBONO}

Sendo o dióxido de carbono um dos principais responsáveis pelo efeito de estufa, a sua captura e o seu armazenamento constituem prioridades da investigação actual. A capacidade de armazenamento deste gás em MOFs depende, tal como no caso do hidrogénio, do seu grau de interacção com os grupos funcionais das redes. Assim, a presença de grupos livres nas pontes orgânicas (como, por exemplo, $-\mathrm{NH}_{2}$ ou $-\mathrm{OH}$ ), de locais de coordenação disponíveis nos centros metálicos, e a natureza dos ligandos orgânicos, ricos em átomos de azoto, potenciam a adsorção selectiva de dióxido de carbono. Vários grupos de investigação focaram a sua actividade nesta área de aplicação dos MOFs sendo um dos materiais mais activos na sequestração de dióxido de carbono oriundo do laboratório de Yaghi: Mg-MOF-74 [52]. Para misturas de $\mathrm{CH}_{4} / \mathrm{CO}_{2}$ (4:1) este material é muito selectivo para o $\mathrm{CO}_{2}$, exibindo uma capacidade de adsorção de 8,8\% em massa. É, também, importante o facto deste gás poder ser libertado do MOF a temperaturas baixas, inferiores a $80{ }^{\circ} \mathrm{C}$.

\subsection{Catálise Heterogénea}

Os catalisadores heterogéneos nanoporosos mais bem sucedidos são, provavelmente, os zeólitos. No entanto, a possibilidade de modificar quimicamente as pontes (ligandos orgânicos) dos MOFs, após a síntese destes permite desenhar novos catalisadores heterogéneos capazes de colmatar algumas das limitações dos zeólitos, nomeadamente obter materiais para catálise enantiosselectiva. Um caso de sucesso descreve a preparação de MOFs quirais capazes de catalisar substratos na reacção aldólica de Mukaiyama [53].

\section{MOFs em Portugal}

Apresenta-se de seguida uma breve resenha da actividade dos grupos de investigação nacionais com interesses 
na área dos MOFs, baseada em uma pesquisa na base de dados da Thomson Reuters Web of Science. O curto espaço aqui disponível não nos permite ser exaustivos, sendo dada ênfase aos estudos levados a cabo na Universidade de Aveiro (UA).

\subsection{AveIRO}

A primeira publicação nacional da síntese de um MOF data de 2002, sendo oriunda do Departamento de Química da UA. Este trabalho descreve uma rede duplamente interpenetrada em que os iões $\mathrm{Cd}^{2+}$ se ligam a dois tipos de ligandos, um deles baseado em ácidos carboxílicos ( $\mathrm{H}_{2}$ bpdc = ácido bifenilo-4,4'-dicarboxílico), o outro em anéis piridínicos (bpe = 1,2-bis(4-piridil)etano), [Cd(bpdc) (bpe)] $\left(\mathrm{H}_{2} \mathrm{O}\right)$ [54]. Desde então, a UA tem liderado em Portugal a investigação nesta área com cerca de 160 publicações, até à data, versando: síntese de novos MOFs; estudos téoricos de adsorção; caracterização estrutural por difracção de raios X (monocristal e pós); aplicações como emissores de luz em sensores, e em catálise.

A investigação em MOFs na UA tem privilegiado o uso de iões lantanídeos e ligandos baseados em grupos fosfonato, visando desenvolver novos materiais funcionais combinando no mesmo material a emissão de luz e a actividade catalítica. Ligandos muito flexíveis, tais como os ácidos $N$-(carboximetil)iminodi(metilfosfónico) $\left(\mathrm{H}_{5} \mathrm{cmp}\right)$ e nitrilotris(metilenofosfónico) $\left(\mathrm{H}_{6} \mathrm{nmp}\right)$ deram origem a várias séries de novos materiais microcristalinos lamelares (2D): [ $\left.\mathrm{Ln}\left(\mathrm{H}_{3} \mathrm{nmp}\right)\right] \cdot 1.5\left(\mathrm{H}_{2} \mathrm{O}\right)[55],\left[\mathrm{Ln}\left(\mathrm{H}_{2} \mathrm{cmp}\right)\left(\mathrm{H}_{2} \mathrm{O}\right)\right]$ [56], [ $\left.\mathrm{Ln}\left(\mathrm{H}_{2} \mathrm{cmp}\right)\right] \cdot x \mathrm{H}_{2} \mathrm{O}$ [57], [ $\left.\mathrm{La}\left(\mathrm{H}_{3} \mathrm{nmp}\right)\right]$ e [La(L)] (com $\left.L^{3-}=\left[-\left(\mathrm{PO}_{3} \mathrm{CH}_{2}\right)_{2}(\mathrm{NH})\left(\mathrm{CH}_{2} \mathrm{PO}_{2}\right) \mathrm{O}_{1 / 2}-\right]_{n}^{3 n-}\right)$ [58]. Apesar das propriedades de fotoluminescência destes materiais serem relativamente modestas, alguns MOFs são bastante activos, por exemplo $[\mathrm{La}(L)]$, em reacções de abertura de anéis epóxido [58]. Com ácido etidrónico ( $\mathrm{H}_{5}$ hedp), um bisfosfonato bastante flexível com potenciais aplicações biológicas, observou-se um caso raro de interconversão de redes cristalinas pela simples adição de $\mathrm{HCl}$ ou $\mathrm{NaCl}$ aos sistemas reaccionais (Figura 8). Mais recentemente, demonstrou-se que é possível utilizar MOFs, com o mesmo ligando, como precursores na preparação de estruturas puramente inorgânicas, sendo a quiralidade do material inicial transposta para a rede inorgânica final [59].

Com o intuito de melhorar as propriedades de fotoluminescência, nomeadamente através do chamado "efeito de antena”, ao longo dos últimos anos temos vindo a desenhar e sintetizar novos ligandos orgânicos baseados em grupos fosfonato ligados a anéis aromáticos. Um dos casos de maior sucesso é um derivado da molécula $\mathrm{H}_{6} \mathrm{nmp}$ em que o átomo de azoto central foi substituído por um anel aromático originando o ácido (benzeno-1,3,5-triiltris(metileno)) trifosfónico $\left(\mathrm{H}_{6} \mathrm{bmt}\right)$. Com esta molécula prepararam-se dois tipos de materiais: por síntese hidrotérmica obteve-se uma nova rede 3D zeolítica; por aquecimento assistido por micro-ondas obteve-se um novo material 1D (Figura 9) [61,62]. A inclusão do anel aromático promoveu a formação de materiais muito mais fotoluminescentes, com rendimentos quânticos acima de 40\%. Estes MOFs revelaram-se, também, excelentes catalisadores heterogéneos na abertura de anéis epóxido; em particular o material 1D consegue em 30 minutos uma conversão e selectividade de $100 \%$.

Uma virtualidade importante dos MOFs nanoporosos é a possibilidade de modificar os ligandos-ponte após a síntese do material através de reacção com moléculas orgânicas, aproveitando a difusão destas através dos nanocanais. Na UA usamos esta via para preparar complexos de iões $\mathrm{Ln}^{3+}$, optimizando a sua esfera de coordenação, no sentido de obter, por exemplo, emissores de luz infravermelha [63].

Recentemente temos vindo a explorar o potencial dos MOFs enquanto materiais para sensores. Assim, a combinação de iões $\mathrm{Ln}^{3+}$ e de um certo ligando fluorado hidrofóbico resultou num material nanoporoso capaz de detectar etanol mesmo na presença de água [64]. Um outro exemplo interessante de MOF estudado em Aveiro foi usado no fabrico de um protótipo de sensor de $\mathrm{pH}$ autocalibrado, que opera na gama de interesse biológico $(\mathrm{pH}=5-7,5)$ [65]. Finalmente, a reacção entre iões $\operatorname{Ln}^{3+}\left(\mathrm{Tb}^{3+}\right.$ e $\left.\mathrm{Eu}^{3+}\right)$ e o ligando BDC resultou em nanocristais de um MOF que funciona como um nanotermómetro, permitindo medir a

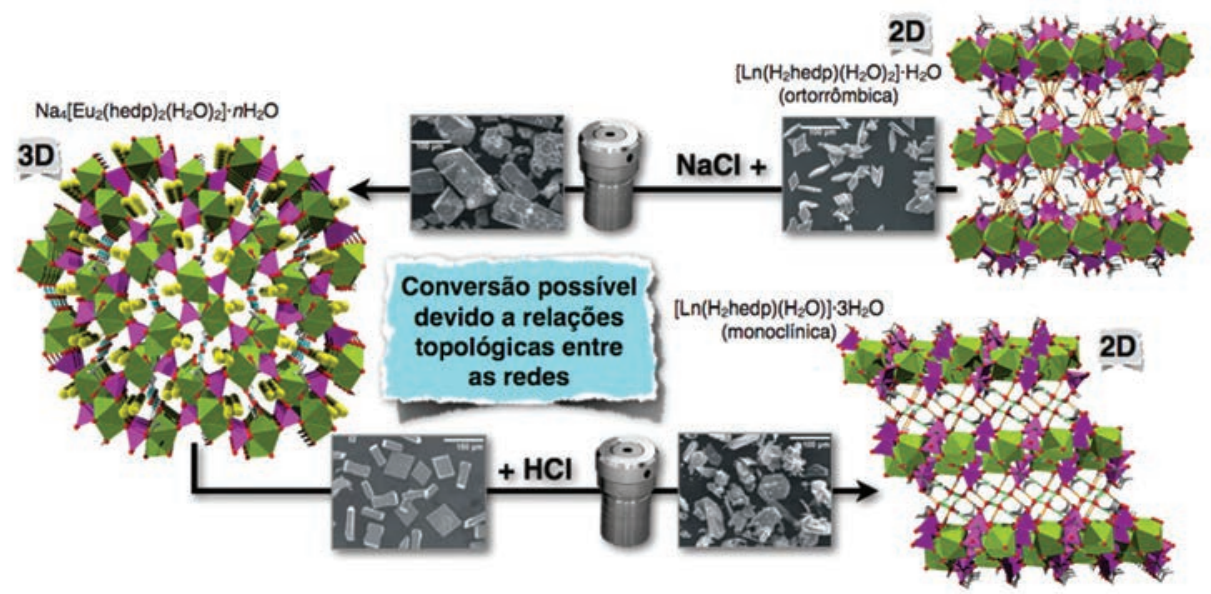

Figura 8 - Interconversão entre redes 2D e 3D de MOFs preparados usando o ácido etidrónico [60] 
temperatura, de forma autocalibrada na gama de temperaturas com relevância biológica (290-320 K) [66]. sosas tais como $\mathrm{CO}_{2} / \mathrm{N}_{2}, \mathrm{CO}_{2} / \mathrm{CH}_{4}$, propano/propileno/isobutano, isómeros derivados do xileno, entre outros. Além

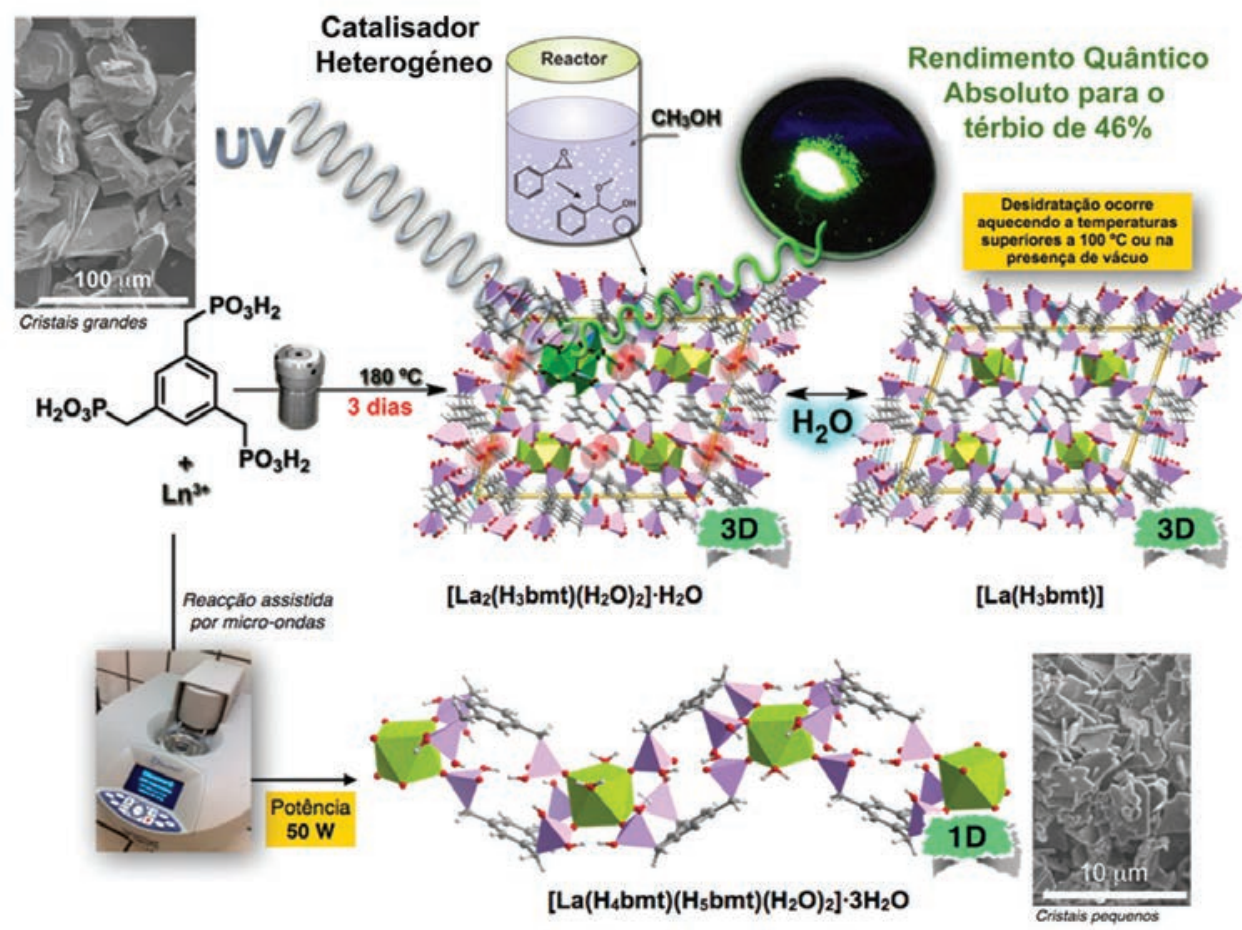

Figura 9 - Diversidade estrutural dos MOFs preparados com o ligando ácido [benzeno-1,3,5-triiltris(metileno)]trifosfónico ( $\mathrm{H}_{6}$ bmt) $[61,62]$

\subsection{LISBOA}

A investigação em MOFs iniciou-se no Instituto Superior Técnico (IST) por volta de 2005, no grupo liderado por Armando Pombeiro. Na última década, este grupo publicou mais de seis dezenas de interessantes artigos científicos na área descrevendo, principalmente, novas estruturas cristalinas contendo metais da primeira série de transição. Em alguns trabalhos, a actividade catalítica de MOFs foi também investigada [67].

Mais recentemente o grupo de investigação de Teresa Duarte (IST) tem focado parte da sua investigação na utilização de certos fármacos (APIs) como ligandos. Um dos exemplos marcantes diz respeito à utilização da gabapentina na preparação de vários MOFs com iões lantanídeo, por métodos de síntese baseados na mecanoquímica [68].

\subsection{PORTO}

Desde 2007 que o grupo coordenado por Alírio Rodrigues (Laboratório Associado LSRE-/LCM, Faculdade de Engenharia da Universidade do Porto) tem liderado em Portugal os estudos de adsorção e separação de gases em MOFs comerciais (por exemplo, HKUST-1) tendo cerca de 30 publicações até à data, muitas delas com grande impacto na comunidade científica da área. Usando conceitos de engenharia química (procurando transpor os estudos da pequena escala até uma escala pré-industrial), este grupo tem-se debruçado sobre os fenómenos da separação de misturas ga- dos estudos experimentais, este grupo tem incluído em muitas das suas publicações cálculos avançados, com vista a explicar em detalhe os resultados obtidos (Figura 10) [69-72].

Mais recentemente, nos Laboratórios do REQUIMTE da Faculdade de Ciências da Universidade do Porto têm sido efectuados estudos de incorporação de polioxometalatos nos canais de MOFs conhecidos, em particular do MIL-101. A formação destes materiais compósitos tem demonstrado potenciar as propriedades catalíticas, aumentando, nomeadamente, a conversão e selectividade [73-74].

\section{CONCLUSÕES}

O intenso esforço de investigação em "Metal-Organic Frameworks" tem confirmado a enorme versatilidade e potencialidade desta área relativamente recente da química de coordenação. Não só é possível inovar do ponto de vista estrutural, com o design e a preparação de novas e exóticas estruturas cristalinas, como é possível explorar as interessantes e úteis propriedades que emergem da combinação de centros metálicos com moléculas orgânicas.

A investigação em Portugal nesta área encontra-se na linha da frente, estando hoje em dia particularmente focada na aplicação dos MOFs como materiais funcionais nas áreas da emissão de luz (fotoluminescência) e dos sensores, adsorção e separação de gases, e catálise. 

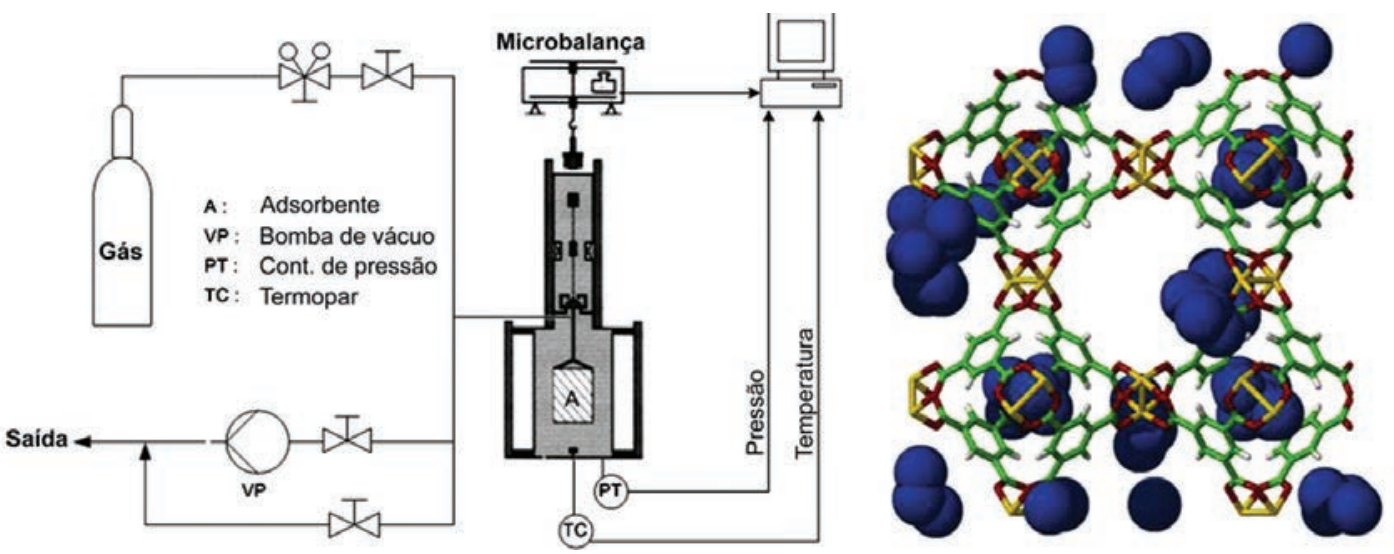

Figura 10 - (Esquerda) Esquema da montagem experimental usada nos estudos de adsorção no Laboratório Associado LSRE-/LCM. (Direita) Representação gráfica da simulação de adsorção de isobutano (a 373K e 3,5 kPa) na estrutura do MOF comercial HKUST-1. Figura adaptada com permissão [70]. (Direitos de autor 2009 Elsevier)

\section{Agradecimentos}

Este trabalho foi financiado por Fundos FEDER através do Programa Operacional Factores de Competitividade - COMPETE, e por Fundos Nacionais através da FCT - Fundação para a Ciência e Tecnologia, no âmbito dos projectos CICECO - FCOMP-01-0124-FEDER-037271 (Ref ${ }^{a}$. FCT PEst-C/CTM/LA0011/2013) e EXPL/CTM-NAN/0013/2013 (FCOMP-01-0124-FEDER-041282). Os autores gostariam ainda de agradecer aos Drs. Patrícia Silva e Sérgio M. F. Vilela pela ajuda prestada na execução gráfica de algumas imagens.

\section{LISTA DE ABREVIATURAS}

1D Unidimensional

2D Bidimensional

3D Tridimensional

API Active Pharmaceutical Ingredient

BDC Benzeno-1,4-dicarboxilato

bpe 1,2-Bis(4-piridil)etano

ELM Elastic Layer-Structured Metal-Organic Framework

$\mathrm{H}_{2}$ bpdc Ácido bifenilo-4,4'-dicarboxílico

$\mathrm{H}_{5} \mathrm{cmp}$ Ácido $\mathrm{N}$-(carboximetil)iminodi(metilfosfónico)

$\mathrm{H}_{5}$ hedp Ácido etidrónico

$\mathrm{H}_{6}$ bmt Ácido [benzeno-1,3,5-triiltris(metileno)]trifosfónico

$\mathrm{H}_{6} \mathrm{nmp}$ Ácido nitrilotris(metilenofosfónico)

HKUST Hong Kong University of Science and Technology

IRMOF Isoreticular Metal-Organic Framework

Ln Lantanídeo

MIL Material from Institute Lavoisier

MOF Metal-Organic Framework

MOP Metal-Organic Polyhedra

MT Metal de transição

NOTT Nottingham

PCN Northwestern University

$\mathrm{UiO} \quad$ University of Oslo

UMCM University of Michigan Crystalline Material
UTSA University of Texas at San António

ZIF Z Zeolitic Imidazolate Framework

\section{REFERÊNCIAS}

[1] F. Wöhler, Annalen der Physik 88 (1828) 253-256

[2] “The Nobel Prize in Chemistry 1913”. Nobelprize.org. Nobel Media AB 2013. Internet: http://www.nobelprize. org/nobel_prizes/chemistry/laureates/1913/ (acedido em 24-07-2014)

[3] http://chem.berkeley.edu/faculty/yaghi/ (acedido em 24-07-2014)

[4] http://www.sbchem.kyoto-u.ac.jp/kitagawa-lab/index-e. html (acedido em 24-07-2014)

[5] http://www.gerard-ferey.org (acedido em 24-07-2014)

[6] H. Li, M. Eddaoudi, M. O’Keeffe, O.M. Yaghi, Nature 402 (1999) 276-279

[7] O.M. Yaghi, M. O’Keeffe, N.W. Ockwig, H.K. Chae, M. Eddaoudi, J. Kim, Nature 423 (2003) 705-714

[8] P. Silva, Lanthanide-Organic Frameworks for Optical and Catalytic Applications, Tese de Doutoramento (2013) Universidade de Aveiro

[9] S.M.F. Vilela, Metal-Organic Frameworks Based on Phosphonate Linkers, Tese de Doutoramento (2013) Universidade de Aveiro

[10] J. Klinowski, F.A.A. Paz, P. Silva, J. Rocha, Dalton Trans. 40 (2011) 321-330

[11] U. Mueller, M. Schubert, F. Teich, H. Puetter, K. SchierleArndt, J. Pastre, J. Mater. Chem. 16 (2006) 626-636

[12] W.J. Son, J. Kim, W.S. Ahn, Chem. Commun. (2008) 6336$-6338$

[13] I. Imaz, D. Maspoch, C. Rodriguez-Blanco, J.M. PerezFalcon, J. Campo, D. Ruiz-Molina, Angew. Chem. Int. Ed. 47 (2008) 1857-1860

[14] T. Friscic, I. Halasz, V. Strukil, M. Eckert-Maksic, R.E. Dinnebier, Croat. Chem. Acta 85 (2012) 367-378

[15] A. Carne-Sanchez, I. Imaz, M. Cano-Sarabia, D. Maspoch, Nat. Chem. 5 (2013) 203-211 
[16] S. Ye, X. Jiang, L.W. Ruan, B. Liu, Y.M. Wang, J.F. Zhu, L.G. Qiu, Microporous Mesoporous Mater. 179 (2013) 191-197

[17] C.E. Wilmer, O.K. Farha, T. Yildirim, I. Eryazici, V. Krungleviciute, A.A. Sarjeant, R.Q. Snurr, J.T. Hupp, Energy Environ. Sci. 6 (2013) 1158-1163

[18] C.H. Lau, R. Babarao, M.R. Hill, Chem. Commun. 49 (2013) 3634-3636

[19] H. Furukawa, K.E. Cordova, M. O’Keeffe, O.M. Yaghi, Science 341 (2013) 974-986

[20] W. Bury, D. Fairen-Jimenez, M.B. Lalonde, R.Q. Snurr, O.K. Farha, J.T. Hupp, Chem. Mat. 25 (2013) 739-744

[21] H.X. Deng, S. Grunder, K.E. Cordova, C. Valente, H. Furukawa, M. Hmadeh, F. Gandara, A.C. Whalley, Z. Liu, S. Asahina, H. Kazumori, M. O’Keeffe, O. Terasaki, J.F. Stoddart, O.M. Yaghi, Science 336 (2012) 1018-1023

[22] F. Millange, R. El Osta, M.E. Medina, R.I. Walton, Crystengcomm 13 (2011) 103-108

[23] Z.Y. Guo, H. Wu, G. Srinivas, Y.M. Zhou, S.C. Xiang, Z.X. Chen, Y.T. Yang, W. Zhou, M. O’Keeffe, B.L. Chen, Angew. Chem. Int. Ed. 50 (2011) 3178-3181

[24] P. Dechambenoit, J.R. Long, Chem. Soc. Rev. 40 (2011) 3249-3265

[25] Y. Yan, I. Telepeni, S.H. Yang, X. Lin, W. Kockelmann, A. Dailly, A.J. Blake, W. Lewis, G.S. Walker, D.R. Allan, S.A. Barnett, N.R. Champness, M. Schröder, J. Am. Chem. Soc. 132 (2010) 4092-4094

[26] M. Klimakow, P. Klobes, A.F. Thunemann, K. Rademann, F. Emmerling, Chem. Mat. 22 (2010) 5216-5221

[27] H. Furukawa, N. Ko, Y.B. Go, N. Aratani, S.B. Choi, E. Choi, A.O. Yazaydin, R.Q. Snurr, M. O’Keeffe, J. Kim, O.M. Yaghi, Science 329 (2010) 424-428

[28] K. Sumida, M.R. Hill, S. Horike, A. Dailly, J.R. Long, J. Am. Chem. Soc. 131 (2009) 15120-15121

[29] D. Saha, S.G. Deng, Int. J. Hydrogen Energy 34 (2009) 2670-2678

[30] K. Koh, A.G. Wong-Foy, A.J. Matzger, J. Am. Chem. Soc. 131 (2009) 4184-4185

[31] S.Q. Ma, D.F. Sun, J.M. Simmons, C.D. Collier, D.Q. Yuan, H.C. Zhou, J. Am. Chem. Soc. 130 (2008) 1012-1016

[32] J.H. Cavka, S. Jakobsen, U. Olsbye, N. Guillou, C. Lamberti, S. Bordiga, K.P. Lillerud, J. Am. Chem. Soc. 130 (2008) 13850-13851

[33] R. Banerjee, A. Phan, B. Wang, C. Knobler, H. Furukawa, M. O’Keeffe, O.M. Yaghi, Science 319 (2008) 939-943

[34] K.S. Park, Z. Ni, A.P. Cote, J.Y. Choi, R.D. Huang, F.J. Uribe-Romo, H.K. Chae, M. O’Keeffe, O.M. Yaghi, Proc. Natl. Acad. Sci. 103 (2006) 10186-10191

[35] A. Kondo, H. Noguchi, S. Ohnishi, H. Kajiro, A. Tohdoh, Y. Hattori, W.C. Xu, H. Tanaka, H. Kanoh, K. Kaneko, Nano Letters 6 (2006) 2581-2584

[36] P.D.C. Dietzel, B. Panella, M. Hirscher, R. Blom, H. Fjellvag, Chem. Commun. (2006) 959-961
[37] O.I. Lebedev, F. Millange, C. Serre, G. Van Tendeloo, G. Férey, Chem. Mat. 17 (2005) 6525-6527

[38] G. Férey, C. Mellot-Draznieks, C. Serre, F. Millange, J. Dutour, S. Surble, I. Margiolaki, Science 309 (2005) 20402042

[39] C. Serre, F. Millange, S. Surble, G. Férey, Angew. Chem. Int. Ed. 43 (2004) 6286-6289

[40] G. Férey, C. Serre, C. Mellot-Draznieks, F. Millange, S. Surble, J. Dutour, I. Margiolaki, Angew. Chem. Int. Edit. 43 (2004) 6296-6301

[41] H.K. Chae, D.Y. Siberio-Perez, J. Kim, Y. Go, M. Eddaoudi, A.J. Matzger, M. O’Keeffe, O.M. Yaghi, Nature 427 (2004) 523-527

[42] C. Serre, F. Millange, C. Thouvenot, M. Nogues, G. Marsolier, D. Louer, G. Férey, J. Am. Chem. Soc. 124 (2002) 13519-13526

[43] S. Onishi, T. Ohmori, T. Ohkubo, H. Noguchi, L. Di, Y. Hanzawa, H. Kanoh, K. Kaneko, Appl. Surf. Sci, 196 (2002) 81-88

[44] K. Barthelet, J. Marrot, D. Riou, G. Férey, Angew. Chem. Int. Ed. 41 (2002) 281-284

[45] D. Li, K. Kaneko, Chem. Phys. Lett. 335 (2001) 50-56

[46] M. Eddaoudi, J. Kim, J.B. Wachter, H.K. Chae, M. O’Keeffe, O.M. Yaghi, J. Am. Chem. Soc. 123 (2001) 43684369

[47] B.L. Chen, M. Eddaoudi, S.T. Hyde, M. O’Keeffe, O.M. Yaghi, Science 291 (2001) 1021-1023

[48] S. Noro, S. Kitagawa, M. Kondo, K. Seki, Angew. Chem. Int. Ed. 39 (2000) 2082-2084

[49] S.S.Y. Chui, S.M.F. Lo, J.P.H. Charmant, A.G. Orpen, I.D. Williams, Science 283 (1999) 1148-1150

[50] Y. Peng, V. Krungleviciute, I. Eryazici, J.T. Hupp, O.K. Farha, T. Yildirim, J. Am. Chem. Soc. 135 (2013) 1188711894

[51] K.L. Mulfort, O.K. Farha, C.L. Stern, A.A. Sarjeant, J.T. Hupp, J. Am. Chem. Soc. 131 (2009) 3866-3868

[52] D. Britt, H. Furukawa, B. Wang, T.G. Glover, O.M. Yaghi, Proc. Natl. Acad. Sci. U. S. A. 106 (2009) 20637-20640

[53] K. Gedrich, M. Heitbaum, A. Notzon, I. Senkovska, R. Frohlich, J. Getzschmann, U. Mueller, F. Glorius, S. Kaskel, Chem. Eur. J. 17 (2011) 2099-2106

[54] F.A.A. Paz, Y.Z. Khimyak, A.D. Bond, J. Rocha, J. Klinowski, Eur. J. Inorg. Chem. (2002) 2823-2828

[55] L. Cunha-Silva, L. Mafra, D. Ananias, L.D. Carlos, J. Rocha, F.A.A. Paz, Chem. Mat. 19 (2007) 3527-3538

[56] L. Cunha-Silva, S. Lima, D. Ananias, P. Silva, L. Mafra, L.D. Carlos, M. Pillinger, A.A. Valente, F.A.A. Paz, J. Rocha, J. Mater. Chem. 19 (2009) 2618-2632

[57] L. Cunha-Silva, D. Ananias, L.D. Carlos, F.A.A. Paz, J. Rocha, Z. Kristall. 224 (2009) 261-272

[58] P. Silva, F. Vieira, A.C. Gomes, D. Ananias, J.A. Fernandes, S.M. Bruno, R. Soares, A.A. Valente, J. Rocha, F.A.A. Paz, J. Am. Chem. Soc. 133 (2011) 15120-15138 
[59] F.N. Shi, F.A.A. Paz, P. Ribeiro-Claro, J. Rocha, Chem. Commun. 49 (2013) 11668-11670

[60] F.N. Shi, L. Cunha-Silva, R.A.S. Ferreira, L. Mafra, T. Trindade, L.D. Carlos, F.A.A. Paz, J. Rocha, J. Am. Chem. Soc. 130 (2008) 150-167

[61] S.M.F. Vilela, A.D.G. Firmino, R.F. Mendes, J.A. Fernandes, D. Ananias, A.A. Valente, H. Ott, L.D. Carlos, J. Rocha, J.P.C. Tomé, F.A.A. Paz, Chem. Commun. 49 (2013) 6400-6402

[62] S.M.F. Vilela, D. Ananias, A.C. Gomes, A.A. Valente, L.D. Carlos, J.A.S. Cavaleiro, J. Rocha, J.P.C. Tomé, F.A.A. Paz, J. Mater. Chem. 22 (2012) 18354-18371

[63] R.M. Abdelhameed, L.D. Carlos, A.M.S. Silva, J. Rocha, Chem. Commun. 49 (2013) 5019-5021

[64] B.V. Harbuzaru, A. Corma, F. Rey, P. Atienzar, J.L. Jordá, H. Garcia, D. Ananias, L.D. Carlos, J. Rocha, Angew. Chem. Int. Ed. 47 (2008) 1080-1083

[65] B.V. Harbuzaru, A. Corma, F. Rey, J.L. Jorda, D. Ananias, L.D. Carlos, J. Rocha, Angew. Chem. Int. Ed. 48 (2009) 6476-6479

[66] C.B. Liu, R.A.S. Ferreira, F.A.A. Paz, A. Cadiau, L.D. Carlos, L.S. Fu, J. Rocha, F.N. Shi, Chem. Commun. 48 (2012) 7964-7966
[67] C. Di Nicola, Y.Y. Karabach, A.M. Kirillov, M. Monari, L. Pandolfo, C. Pettinari, A.J.L. Pombeiro, Inorg. Chem. 46 (2007) 221-230

[68] S. Quaresma, V. André, A.M.M. Antunes, L. Cunha-Silva, M.T. Duarte, Cryst. Growth Des. 13 (2013) 5007-5017

[69] J.W. Yoon, Y.K. Seo, Y.K. Hwang, J.S. Chang, H. Leclerc, S. Wuttke, P. Bazin, A. Vimont, M. Daturi, E. Bloch, P.L. Llewellyn, C. Serre, P. Horcajada, J.M. Greneche, A.E. Rodrigues, G. Férey, Angew. Chem. Int. Ed. 49 (2010) 5949-5952

[70] N. Lamia, M. Jorge, M.A. Granato, F.A.A. Paz, H. Chevreau, A.E. Rodrigues, Chem. Eng. Sci. 64 (2009) 3246-3259

[71] L. Bastin, P.S. Barcia, E.J. Hurtado, J.A.C. Silva, A.E. Rodrigues, B.L. Chen, J. Phys. Chem. C 112 (2008) 1575-1581

[72] M.P.M. Nicolau, P.S. Barcia, J.M. Gallegos, J.A.C. Silva, A.E. Rodrigues, B.L. Chen, J. Phys. Chem. C 113 (2009) 13173-13179

[73] D.M. Fernandes, A.D.S. Barbosa, J. Pires, S.S. Balula, L. Cunha-Silva, C. Freire, ACS Appl. Mater. Interfaces 5 (2013) 13382-13390

[74] C.M. Granadeiro, P. Silva, V.K. Saini, F.A.A. Paz, J. Pires, L. Cunha-Silva, S.S. Balula, Catal. Today 218 (2013) 35-42

\section{Actualidades Cientificas}

\section{CÁLCULOS PREVEÊM NOVA FORMA DE FÓSFORO}

No início do ano ficou-se a saber que é possível obter fosforeno (estrutura bidimensional semelhante ao grafeno) através de um processo de esfoliação do fósforo preto, que possui uma estrutura hexagonal lamelar semelhante à da grafite. Mais recentemente, investigadores da Universidade de Michigan, EUA, previram a existência de fósforo azul, outra forma lamelar, usando cálculos computacionais. Os cálculos sugerem que a nova forma de fósforo terá uma estrutura e propriedades semelhantes à do fósforo preto que poderá ser ideal para uma possível utilização em dispositivos de nanoelectrónica.

O fósforo preto e o denominado fósforo azul são alótropos hexagonais de fósforo feitos de camadas bidimensionais

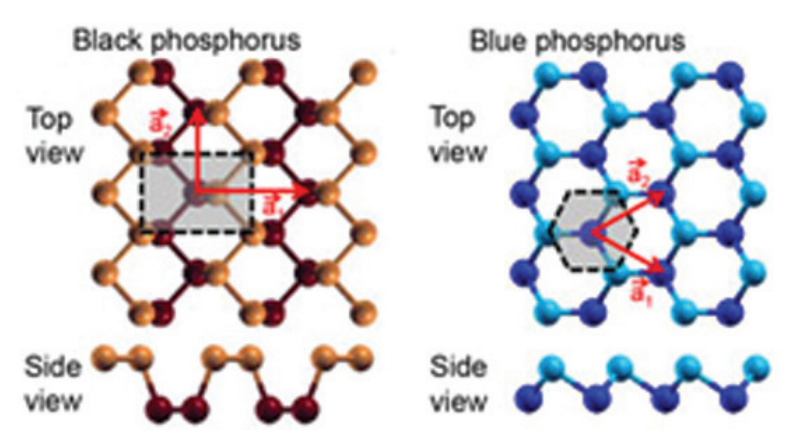
sobrepostas. No entanto, uma das camadas de fósforo azul tem uma maior semelhança com o grafeno, tornando-a ligeiramente mais achatada que a da correspondente de fósforo preto. Os cálculos realizados por Zhen Zhu e David Tomanek mostram que ambas as formas são igualmente estáveis, mas o fósforo azul pode ter melhores propriedades eléctricas o que o torna mais adequado para aplicações em electrónica bidimensional. Tal como o fósforo preto, deverá ser possível esfoliar o fósforo azul para produzir camadas bidimensionais. Esta previsível nova forma alotrópica de fósforo ainda não foi preparada mas os

investigadores sugerem possíveis formas de o fazer.

(fontes: http://www.rsc.org/chemistryworld/2014/04/model-predicts-blue-phosphorus; Z. Zhu, D. Tomanek; Phys. Rev. Lett 112 (2014) DOI: 10.1103/PhysRevLett.112.176802) 


\section{Essential reading for multidisciplinary chemists}

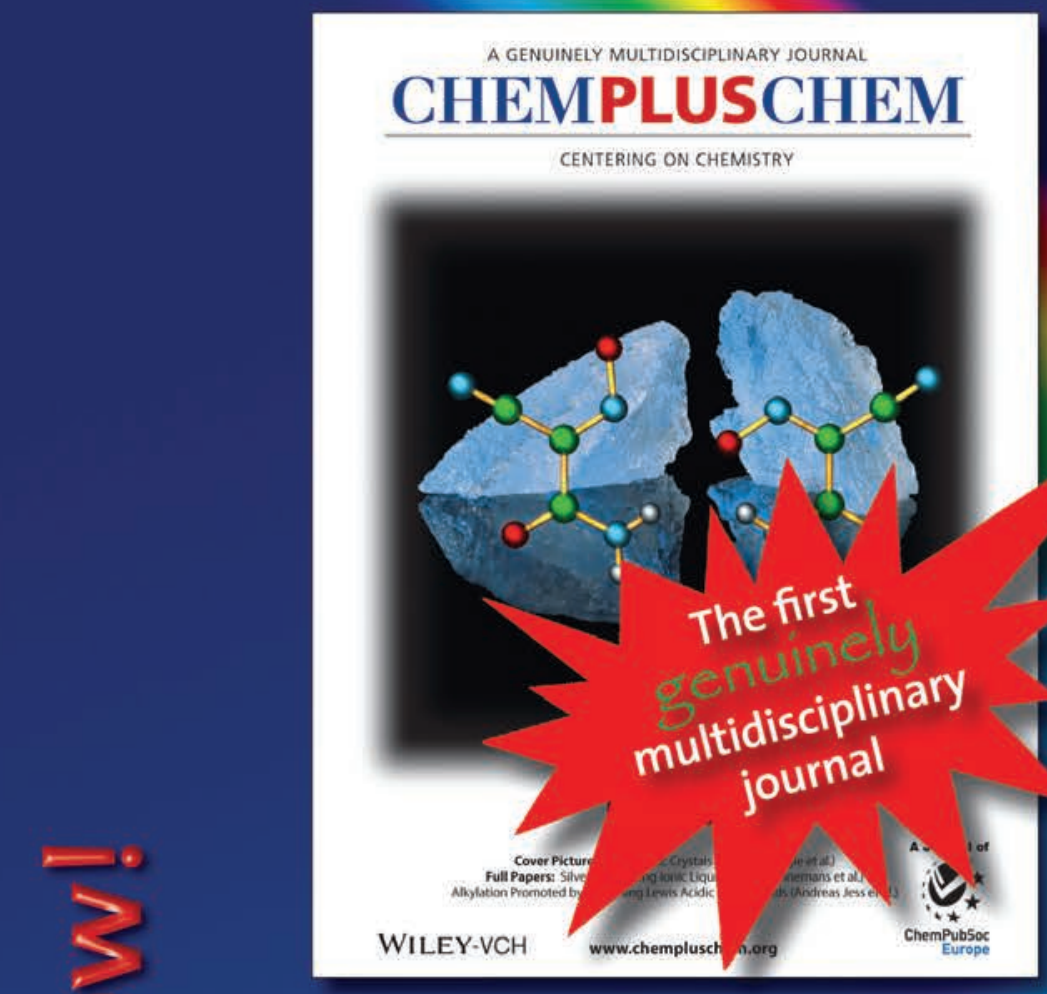

www.chempluschem.org

Editorial Team

Editor: Neville Compton

Deputy Editor: Marisa Spiniello

ChemPlusChem presents multidisciplinary research in a variety of formats:

Minireviews

Reviews

Full Papers

Communications

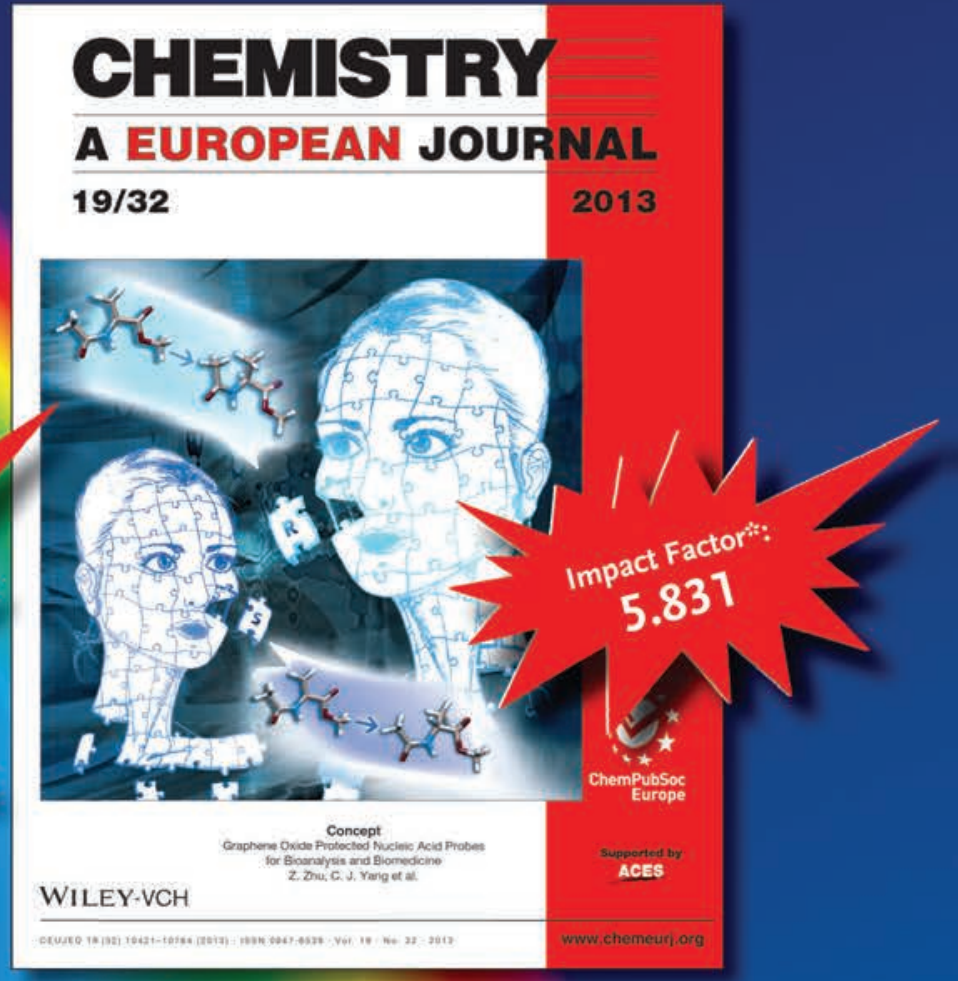

www.chemeurj.org

Editorial Team

Editor: Neville Compton

Deputy Editors: Anne Deveson and Elisabeth Roedern

"2013 Release of Journal Citation Reports"

Source:Thomson Reuters, 2012 Citation Data

ChemPlusChem succeeds the

Collection of Czechoslovak Chemical

Communications

Articles in ChemPlusChem cover at least two different field of chemistry or one of chemistry and one of another scientific discipline:
general chemistry
biotechnology
analytical chemistry
organic chemistry
inorganic chemistry
biological chemistry
materials chemistry
physical chemistry
organometallic chemistry
nanochemistry
macromolecular chemistry
polymer chemistry
medicinal chemistry
electrochemistry
catalysis
computational chemistry
environmental chemistry
coordination chemistry

manuscript) press

A journal of:

Published by:

$\underbrace{\star}_{\star \star}$ ChempubSoc
Europerising
compuropean
chemical societies

WILEY-VCH

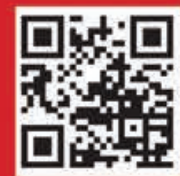

